\title{
Guest editorial on special section: Automotive Software Architecture
}

\author{
Yanja Dajsuren ${ }^{\mathrm{a}, \mathrm{b}}$, Harald Altinger ${ }^{\mathrm{c}}$, Miroslaw Staron ${ }^{\mathrm{d}, \mathrm{e}, *}$ \\ a Eindhoven University of Technology, The Netherlands \\ ${ }^{\mathrm{b}}$ Centrum Wiskunde E Informatica, The Netherlands \\ ${ }^{c}$ AUDI AG, Germany \\ ${ }^{\mathrm{d}}$ Chalmers University of Technology, Sweden \\ e University of Gothenburg, Sweden
}

\section{A R T I C L E I N F O}

\section{Article history:}

Received 9 November 2015

Revised 12 November 2015

Accepted 12 November 2015

Available online $\mathrm{xxx}$

A modern day vehicle might be seen as a complex system containing millions of lines of code running on multiple microcontrollers creating a heterogeneous, deeply coupled networking system. Nowadays trends like V2X (Vehicle to Vehicle, Vehicle to Infrastructure, Vehicle to Pedestrian), electric vehicles or self-driving cars are all based on software and electronics. These new features require new engineering approaches and more advanced software architectures suitable for the automotive domain. The alignment of the architectural, design, and engineering concepts are in urgent need given the ever increasing software complexity and size. Furthermore, up to $90 \%$ of the innovation in modern cars are enabled by software, thus, more and more innovations (e.g. augmented reality, connectivity, and Internet of Things) need more sophisticated and reliable approaches from the software architecture and component-based software engineering community.

Therefore, to bring together researchers and practitioners in the area of automotive software engineering, the international Workshop on Automotive Software Architectures (WASA) was held on May 4, 2015 in Montréal, Canada and co-located with the 12th Working IEEE/IFIP Conference on Software Architecture (WICSA) and the 9th CompArch federated conference series, the premier gatherings of software architecture and component-based software engineering practitioners and researchers. The objective of the workshop is to become the premier forum for presentation of research results, industrial experience reports, and future trend discussions on the automotive software architecture field.

WASA gives researchers and practitioners a unique opportunity to share their perspectives with others interested in the various aspects of the automotive software architecture field. The first edition of

\footnotetext{
* Corresponding author at: University of Gothenburg, Sweden . Tel.: +46317721081.

E-mail address: miroslaw.staron@gu.se (M. Staron).
}

WASA covered a variety of topics such as automotive system/software architecture, AUTOSAR, automotive software quality, safety, and testing. The call for papers attracted submissions from Europe, Canada, and Brazil. Five full papers, four position or future trend papers, and one industrial paper were submitted. From these 10 submissions, 7 papers were accepted to be presented at the workshop - $80 \%$ of full papers, $50 \%$ of position or future trend papers, and $100 \%$ of industrial papers. The proceedings of the WASA'15 workshop was published by the ACM - http://dl.acm.org/citation.cfm?id=2752489, as the WICSA/CompArch companion proceedings. The WASA'15 workshop website is located at http://www.win.tue.nl/wasa2015/.

It was one full-day workshop with an appealing program thanks to our keynote speaker and all the presenters who shared their research results and industrial experiences. The program comprised of four sessions - Keynote address, Automotive architecture and standards, Automotive architecture and quality, and Panel discussion. The keynote was given by Prof. Dr. James Cordy (Queen's University, Canada) and was titled "SIMONE: Architecture-Sensitive Near-miss Clone Detection for Simulink Models". The talk outlined the challenges of Simulink model clone detection and presented the design and implementation of SIMONE clone detection tool. SIMONE was demonstrated on complex Simulink models from the automotive industry.

The automotive architecture and standards session consisted of the following presentations:

- A functional architecture for autonomous driving;

- Functional safety measurement in the automotive domain: adaptation of PSM;

- A survey on the benefits and drawbacks of AUTOSAR.

The automotive architecture and quality session consisted of the following presentations: 
- Metrics for verification and validation of architecture in powertrain software development;

- Automatic consequence analysis of automotive standards (AUTOCAAS);

- Challenges from integration testing using interconnected hardware-in-the-loop test rigs at an automotive OEM - an industrial experience report;

- Reliability analysis and quality impact prediction in application architecture evolution.

In the panel discussion we invited all authors and the keynote speaker to present their view on the following topics:

- What are the most important current trends in automotive software architecture?

- How do the automotive standards like AUTOSAR, ISO 26262 influence the architectures?

- Which open research directions should be prioritized?

- How can the automotive aspects of architecting be highlighted in the software architecture community at large?

The most important trends were identified to be the disruptive technologies in the automotive sector - new companies entering the market and shifting the focus from building cars to building connected products. Examples of such trends are the self-driving car of Google or Tesla's electrical cars. AUTOSAR was identified as one of the trends changing the way that the automakers treat cars - treating the cars as platforms which can include application code with varying functionality - a statement that concluded the discussion.

For this special section of Elsevier's Information and Software Technology journal, two papers were selected from the WASA'15 papers based on the best reviewing score and positive comments by the program committee members and workshop chairs. These two papers are - "A functional architecture for autonomous driving" and "Functional safety measurement in the automotive domain: adaptation of PSM". Authors added more than 40\% new materials to the workshop papers. The papers were reviewed by 6 domain experts over a period of 3 months. We are pleased to present these high quality and insightful research results to the research community.
The paper by Sagar Beherea and Martin Törngren proposes a functional reference architecture for an autonomous driving vehicle based on the cumulative research results and practical development of building prototype autonomous vehicles over a period of five years. They identify and divide functional components into three main categories - Perception, Decision and Control, and Vehicle platform manipulation. Two alternative solutions present different ways to distribute the functional components across the vehicle platform and cognitive driving intelligence layers. Based on the stakeholder requirements and analysis of alternative solutions, a functional reference architecture is proposed and applied to different vehicles such as commercial trucks and passenger cars.

The paper by Yaping Luo and Mark van den Brand presents a study on metrics design for safety assessment. They identified three sources for gathering metrics information: industrial interests, safety standards, and available data. For each source, different methods have been proposed and used for obtaining and designing the relevant metrics. They applied their methods in a European project OPENCOSS, where the obtained relevant metrics have also been validated via a survey.

Putting together WASA'15 was a team effort. First we would like to thank all the authors for submitting articles and providing the content of the program. We are grateful to the program committee and the journal's anonymous reviewers, who worked very hard in reviewing papers and providing feedback for authors. We would like to thank the Editor-in-Chief Prof. Dr. Claes Wohlin for his support and guidance throughout the preparation of this special section. Furthermore our thanks go to the keynote speaker Prof. Dr. James Cordy, who shared valuable and inspiring results to his peers. Finally, we thank the hosting university, our supporters, Software Center and the journal of Information and Software Technology.

The second edition of WASA is going to be organized with the 13th Working IEEE/IFIP Conference on Software Architecture (WICSA) and the 10th CompArch federated conference series. It will take place in Venice, Italy during April 5-8, 2016. For the call for papers and further information, visit WASA 2016 website: http://www.win.tue.nl/ wasa2016/. 